\title{
Editorial
}

\section{Identifying failure to achieve complete (TIMI 3) reperfusion following thrombolytic treatment: how to do it, when to do it, and why it's worth doing}

The TIMI scoring system has been used extensively to report infarct related artery patency in trials of thrombolytic treatment. In the initial studies no distinction was made between TIMI 2 and 3 flow, both being considered to represent an open artery. Karagounis and colleagues reported data from the TEAM-2 study in 1992 suggesting that the outcomes of patients with TIMI 2 flow were closer to those of patients with no reperfusion (TIMI 0/1) than they were to patients with "complete" reperfusion (TIMI 3). ${ }^{1}$ The GUSTO angiographic substudy reported TIMI 2 and TIMI 3 flow as separate groups; 30 day mortality was $8.9 \%$ for patients with TIMI $0 / 1$ flow at 90 minute angiography compared to $7.4 \%$ for TIMI 2 flow and $4.4 \%$ for TIMI 3 flow. The size of the study precluded this impressive $40 \%$ relative reduction in mortality between TIMI 2 and 3 from reaching significance $(p=0.08)$, but left ventricular function was significantly better in patients achieving TIMI 3 flow compared to TIMI 2 flow. ${ }^{2}$ If we accept that the goal of treatment in acute myocardial infarction is to achieve complete (TIMI 3) reperfusion then it becomes critically important to be able to identify failure to achieve TIMI 3 flow simply and rapidly so that additional treatment strategies or randomisation in appropriate trials can be considered. Two papers in this issue of Heart describe similar methods of assessing reperfusion and importantly report patients with TIMI 2 and 3 flow as separate groups. ${ }^{34}$

\section{Clinical markers of reperfusion}

Using the persistence or resolution of chest pain as a guide to reperfusion is complicated by the clinical requirement to relieve pain adequately, and by significant inter-individual variations in pain threshold and the release of endogenous opioid-like peptides. In the TAMI study, $60 \%$ of the subgroup with no change in their chest pain before angiography had a patent artery (reported as TIMI $2 / 3$ ). ${ }^{5}$ Oude Ophuis and colleagues have assessed the change in intensity of chest pain in an observational study of 230 patients undergoing coronary angiography at a median of 73 minutes following hospital admission with acute myocardial infarction. ${ }^{3}$ Only $72 \%$ of this group received a thrombolytic drug, but overall a sudden decrease in chest pain occurred in $36 \%$ of the patients and was more frequent than any of the five electrocardiographic parameters assessed. A sudden decrease in chest pain had a positive predictive value of $57 \%$ for TIMI 3 flow as opposed to TIMI $0-2$ flow. The negative predictive accuracy was higher at $86 \% .^{3}$

Electrocardiographic markers of reperfusion Previous studies looking at the relation between changes in paired standard 12 lead ECGs, arrhythmias, and angi- ographically assessed reperfusion following thrombolytic treatment have generally reported patients with TIMI 2 and 3 flow as a single group. These studies have suggested that the best marker of reperfusion is a $>50 \%$ decrease in ST segment elevation in the single lead with the maximum ST elevation at presentation. ${ }^{6}$ Oude Ophuis and colleagues have confirmed that this parameter had the highest positive predictive accuracy of their five reported ECG/arrhythmia criteria for TIMI 3 flow versus TIMI 0-2 flow; however, the value obtained was only $66 \%$ with a higher negative predictive accuracy of $86 \%$. If four or more clinical/ECG criteria for reperfusion were present, this conferred a $99 \%$ specificity for TIMI 3 flow with an $80 \%$ positive and $73 \%$ negative predictive accuracy. ${ }^{3}$ In the study of Sutton and colleagues a consecutive series of 99 patients with acute myocardial infarction received thrombolytic treatment with streptokinase. Six different ECG/arrhythmia criteria were assessed; the combination of a $<50 \%$ decrease in the ST elevation in the lead with the maximum ST elevation coupled with the absence of any accelerated idioventricular rhythm predicted absence of TIMI 3 flow at two hours post-thrombolysis, with a sensitivity and specificity of $81 \%$ and $88 \%$, respectively, and a positive and negative predictive accuracy of $87 \%$ and $83 \%$, respectively. ${ }^{4}$ Continuous on-line 12 lead ST segment and/or vectorcardiographic monitoring may facilitate the identification of failed reperfusion. ${ }^{78}$ In particular, if the QRS and ST vector traces cross over this probably does represent myocardial reperfusion. Both techniques require careful attention to detail to avoid contamination by artefact, but can be incorporated into a coronary care unit monitoring system.

\section{Biochemical markers of reperfusion}

Serum concentrations of total creatine kinase (CK), $\mathrm{CK}-\mathrm{MB}$, myoglobin, and both isoforms of cardiac troponin have been studied separately and in combination to assess their ability to identify reperfusion following thrombolytic treatment. The largest individual study comes from the TIMI 10B investigators and compared the ability of myoglobin, CK-MB, and cardiac troponin-I to predict infarct artery patency assessed at 60 minutes following administration of TNK-tPA (tenecteplase) in 442 patients with acute myocardial infarction. The ratio of the 60 minute to baseline concentration provided the best separation between TIMI $0 / 1$ and TIMI $2 / 3$, with no significant difference in the performance of each of the three serum markers. For example, a 60 minute myoglobin ratio of $\geqslant 4.0$ predicted a $90 \%$ probability of TIMI $2 / 3$ flow. Unfortunately, none of the markers or derived values were particularly good at separating patients with TIMI 3 from those with TIMI 0-2 flow, with the 60 minute myoglobin ratio having a positive predictive value of $59 \% .{ }^{9}$ A review of 
biochemical markers of reperfusion for the International Federation of Clinical Chemistry (IFCC) committee on standardisation of markers of cardiac damage has also concluded that there are no serum markers currently available which can reliably differentiate between TIMI 2 and TIMI 3 flow. ${ }^{10}$ Nevertheless, these markers are now available as rapid near-patient assays ideal for use in the coronary care unit and, when used in conjunction with ECG and clinical markers, may contribute to a decision algorithm to guide a decision to proceed with further reperfusion treatment.

\section{When to assess reperfusion}

Most studies have assessed infarct related artery patency at 90 minutes, with some recent trials also including 60 minute angiography to look for earlier reperfusion with newer single bolus thrombolytic drugs. The data of Oude Ophuis and colleagues were collected at a median of 73 minutes from admission, and the needle to catheter laboratory time in the thrombolytic subgroup must have been 15-20 minutes less than this. Accordingly their reperfusion criteria have been derived earlier than most other groups; indeed, the TIMI $0 / 1$ rate was high at $57 \%$, albeit that $28 \%$ of their patients had not received any reperfusion treatment at the time of angiography. ${ }^{3}$ This strategy resulted in a very high rate of percutaneous transluminal coronary angioplasty (PTCA) (47\% in their study), but it may nevertheless minimise the time to reperfusion and the one year mortality for all 230 patients was low at $6.5 \%$. Sutton and colleagues took the long view and waited for two hours post-thrombolysis to assess reperfusion. The rationale here is to avoid an unnecessary trip to the catheter laboratory for the patient and the on-call team, and indeed they report a very respectable TIMI 3 flow rate of 59/99 patients postangiography (increased to 89/99 post-rescue PTCA). ${ }^{4}$ The provision of an evidence base in this area would require a trial with clinical end points in which patients were randomly assigned to further reperfusion treatment according to agreed criteria of failed reperfusion at different time points post-thrombolysis.

\section{Why it's worth doing}

The nihilist's view might be that there is no convincing evidence to support the use of either rescue thrombolysis or rescue PTCA in patients with failed reperfusion, and so why bother to try to identify it.

The "RESCUE" trial is the largest randomised trial of rescue PTCA, and recruited 151 patients with TIMI 0/1 flow in the infarct related artery $<8$ hours from the onset of chest pain. It showed a trend towards lower mortality and less heart failure which almost reached significance. ${ }^{11}$ This trial did not recruit patients with TIMI 2 flow and it was conducted in the pre-stent/IIb/IIIa blocker era. Subgroup analysis in TIMI 4 and TAMI clearly confirmed that rescue PTCA could convert the majority of patients with < TIMI 3 flow to TIMI 3 even without the use of stents and IIb/IIIa blockers, but there was a high reocclusion rate and a very high mortality in patients with failed rescue PTCA. ${ }^{12}{ }^{13}$ In a more contemporary population of patients undergoing rescue PTCA reported in this issue of Heart, the procedural success rate was $87 \%$ and the reinfarction rate was low at $1.9 \%$, possibly because of a relatively high use of stents (55\%) and abciximab (21\%). In addition, although patients with cardiogenic shock were excluded from this series, $36 \%$ of patients received intra-aortic balloon pump counterpulsation and this may have favourably influenced the rate of reocclusion. Nevertheless a high in-hospital mortality (43\%) in failed rescue PTCA was again observed. ${ }^{14}$ Some regional catheterisation laboratories have developed and reported the results of a policy of administering thrombolytic treatment in the local hospital, with immediate transfer to the invasive centre for intentional rescue PTCA. This strategy has the obvious advantage of minimising the delay between presentation and rescue PTCA, but large numbers of patients are transferred and subsequently require no intervention. In one series $35 \%$ of transferred patients ended up having rescue PTCA with a high success rate $(96 \%)$ and low incidence of reinfarction $(2 \%) .^{15}$

\section{Even TIMI 3 flow may not be good enough?}

Some patients with < TIMI 3 flow have impaired myocardial perfusion because of early no-reflow caused by platelet/thrombus microembolisation, and are unlikely to be helped by rescue PTCA. Doppler flowire and myocardial contrast echo studies have confirmed that this also applies to a subgroup of patients with TIMI 3 flow. ${ }^{16}{ }^{17}$ The differentiation of this situation from one in which there is a flow limiting angiographically obscure dissection is sometimes difficult and may require intravascular ultrasound. Empirical stent implantation in this scenario can result in a reduction in flow presumably caused by further embolisation of the microcirculation. One of the hypotheses being tested in the ongoing GUSTO 4 study is that the combination of abciximab and thrombolytic treatment will improve clinical outcomes by reducing the incidence of no-reflow both following initial treatment and in the context of any rescue PTCA subsequently required. In the study of Sutton and colleagues, the 59 patients with TIMI 3 flow at two hours post-thrombolysis had a high early ( $<10$ days) adverse event rate with $15 / 59$ (25\%) experiencing either reinfarction (11) or unstable angina (4) requiring PTCA, and an additional six patients undergoing CABG during the index admission. ${ }^{4}$ The TIMI 3 flow rate in these patients at 90 minutes was not known, but failure to reperfuse by 90 minutes may predict a poor outcome even if there is subsequently reperfusion between 90 and 120 mins; such delayed reperfusion, in addition to being associated with more extensive infarction, may reflect complex lesion morphology with a severe residual stenosis and/or large thrombus burden and consequently a high rate of recurrent ischaemia/infarction. In addition, there was only one immediate PTCA in this group, and although the results of immediate PTCA in the pre-stent/IIb/IIIa blocker era were poor, this is another area which may now have to be revisited.

\section{Summary}

In routine practice, waiting until two hours postthrombolysis to make a decision to proceed to rescue PTCA would usually entail a further delay of 30-60 minutes while the on-call team arrive and set up, or sometimes even longer if there is an ongoing procedure in the laboratory(ies). In addition, it has been shown that clinical outcome can be just as accurately predicted by the change in ST segment elevation following thrombolysis at $60 \mathrm{~min}-$ utes as by later time points. ${ }^{18}$ Accordingly, in the case of a unit with a policy of rescue PTCA for failed reperfusion, the patient and the 12 lead ECG should be assessed at 60-90 minutes with a view to either calling out the catheterisation laboratory team or, if appropriate, entering the patient into a relevant study. In centres without on-site PTCA, rescue thrombolysis can be considered or the patient can be transferred to a PTCA centre. The option of transferring all patients immediately after initiation of thrombolytic treatment is unlikely to be feasible in the UK. A definitive assessment of the value of rescue thrombolysis and rescue PTCA should be provided by the ongoing British Heart Foundation sponsored REACT trial in which patients diagnosed to have failed reperfusion are 
randomised to either intravenous heparin for 48 hours, rescue thrombolysis or rescue PTCA, with a requirement that further reperfusion treatment must be delivered within 12 hours of the onset of chest pain. Patients can be randomised if there is a $<50 \%$ decrease in ST segment elevation in the lead with the maximum ST segment elevation before thrombolysis. This assessment is made at 90 minutes after thrombolytic treatment, and although it is reassuring that the utility of this non-invasive marker has been reconfirmed, it will still incorrectly categorise a substantial minority. Faced with a patient having an extensive myocardial infarction in whom there is doubt as to the adequacy of reperfusion, coronary angiography does not seem unreasonable.

Lanarkshire Cardiac Catheterisation Laboratory,

K G OLDROYD

Hairmyres Hospital,

East Kilbride,

G75 8RG, UK

1 Karagounis L, Sorensen SG, Menlove RL, et al. Does thrombolysis in myocardial infarction (TIMI) perfusion grade 2 represent a mostly patent artery or a mostly occluded artery? Enzymatic and electrocardiographic evidence from the TEAM-2 study. Second multicenter thrombolysis trial of eminase from the TEAM-2 study. Second multicenter thrombolysis trial of

2 GUSTO Angiographic Investigators. The effects of tissue plasminogen activator, streptokinase or both on coronary-artery patency, ventricular function, and survival after acute myocardial infarction. $N$ Engl $\mathcal{F} \mathrm{Med}$ 1993;329:1615-22.

3 Oude Ophuis AJ, Bär FW, Vermeer F, et al. Angiographic assessment of prospectively determined non-invasive reperfusion parameters in acute myocardial infarction. Heart 2000;84:164-70.

4 Sutton AGC, Campbell PG, Price DJA, et al. Failure of thrombolysis by streptokinase: detection with a simple electrocardiographic method. Heart 2000;84:149-56.

5 Califf RM, O'Neill W, Stack RS, et al. Failure of simple clinical measurements to predict perfusion status after intravenous thrombolysis. Ann Intern Med 1988;108:658-62.

6 Hogg KJ, Hornung RS, Howie CA, et al. Electrocardiographic prediction of coronary artery patency after thrombolytic treatment in acute myocardial
infarction: use of ST-segment as a non-invasive marker. Br Heart $f$ 1988;60:275-80.

7 Langer A, Krucoff MW, Klootwijk P, et al. Noninvasive assessment of speed and stability of infarct-related artery reperfusion: results of the GUSTO ST segment monitoring study. Global utilization of streptokinase and tissue plasminogen activator for occluded coronary arteries. $\mathcal{F}$ Am Coll Cardiol 1995;25:1552-7.
GUSTO, Global Utilization of Streptokinase and t-PA for Occluded coronary arteries

REACT, Rescue Angioplasty vs Conservative Treatment or Repeat Thrombolysis

TAMI, Thrombolysis and Angioplasty in Myocardial Infarction

TEAM, Trial of Eminase in Acute Myocardial Infarction

TIMI, Thrombolysis in Myocardial Infarction

8 Juhlin P, Bostrom PA, Hansen O, et al. Continuous vectorcardiographic changes in relation to scintigraphic signs of reperfusion in patients with acute myocardial infarction receiving thrombolytic therapy. $f$ Intern Med 1996;239:35-41.

9 Tanasijevic MT, Cannon CP, Antman EM, et al for the TIMI 10B Investigators. Myoglobin, creatine-kinase-MB and cardiac troponin-I 60 minute ratios predict infarct-related artery patency after thrombolysis for acute myocardial infarction. F Am Coll Cardiol 1999;34:739-47.

10 Apple FS. Biochemical markers of thrombolytic success. Scand f Clin Lab Invest 1999;59(suppl 230):60-6.

11 Ellis SG, Ribeiro da silva E, Heyndrickx G, et al for the RESCUE Investigators. Randomized comparison of rescue angioplasty with conservative management of patients with early failure of thrombolysis for acute anterior management of patients with early failure of thromb
myocardial infarction. Circulation 1994;90:2280-4.

12 Gibson CM, Cannon CP, Greene RM, et al for the TIMI 4 Study Group. Rescue angioplasty in the thrombolysis in myocardial infarction (TIMI) 4 Rescue angioplasty in the thromb
trial. Am f Cardiol 1997;80:21-6.

13 Ward SR, Sutton JM, Pieper KS, et al for the TAMI Investigators. Effects of thrombolytic regimen, early catheterization, and pre-discharge angiographic variables on six-week left ventricular function. Am f Cardiol 1997; 79:539-44.

14 Sutton AGC, Campbell PG, Grech ED, et al. Failure of thrombolysisexperience with a policy of early angiography and rescue angioplasty for electrocardiographic evidence of failed thrombolysis. Heart 2000;84:197204.

15 Oude Ophuis TJ, Bär FW, Vermeer F, et al. Early referral for intentional rescue PTCA after initiation of thrombolytic therapy in patients admitted to a community hospital because of a large acute myocardial infarction. Am Heart f 1999;137:846-53.

16 Ito $\mathrm{H}$, Maruyama A, Iwakura K, et al. Clinical implications of "no-reflow" phenomenon: a predictor of complications and left ventricular remodeling in reperfused anterior wall myocardial infarction. Circulation 1996;93:2238 .

17 Iwakura $\mathrm{K}$, Ito $\mathrm{H}$, Takiuchi S, et al. Alternation in the coronary blood flow velocity pattern in patients with no reflow and reperfused acute myocardial infarction. Circulation 1996;94:1269-75.

18 Purcell IF, Newall N, Farrer M. Change in ST segment elevation 60 minutes after thrombolytic initiation predicts clinical outcome as accurately as later electrocardiographic changes. Heart 1997;78:465-71.

\section{STAMPS IN CARDIOLOGY}

\section{Institutions}

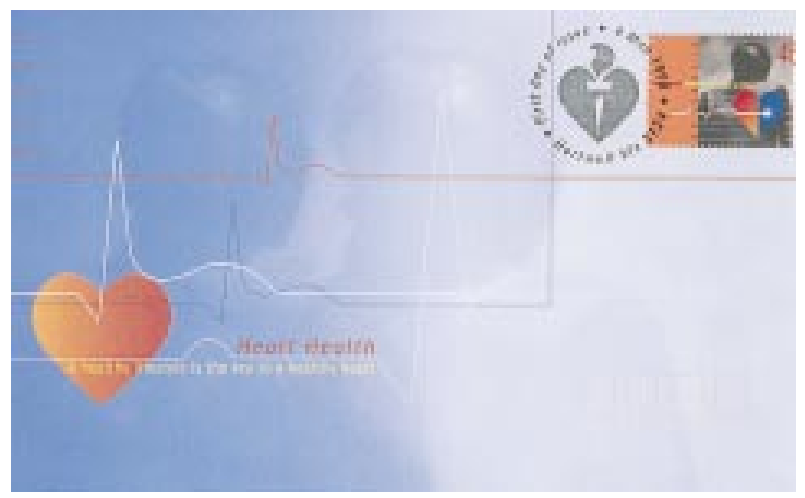

This 45 cent Australian stamp was released on 4th May 1998 marking the National Heart Foundation's Heart Week from 3-10th May. The stamp is inscribed "Heart attack claims one life every 20 minutes". A miniature sheet of 10 of these stamps was also issued with the telephone number of the National Heart Foundation Information Service incorporated in the border. The ECG with ST segment elevation is a key feature of the First Day Cover which also contains public information on the prevalence of cardiovascular disease and risk factors on the reverse.

M K DAVIES A HOLLMAN 